\title{
Citrate capped superparamagnetic iron oxide nanoparticles used for hyperthermia therapy
}

\author{
Elham Cheraghipour ${ }^{1}$, Sirus Javadpour ${ }^{1}$, Ali Reza Mehdizadeh ${ }^{2}$ \\ ${ }^{1}$ Department of Materials Science and Engineering, Shiraz University, Shiraz, Iran \\ ${ }^{2}$ Department of Medical Physics, Shiraz University of Medical Sciences, Shiraz, Iran \\ Email: elham.cheraghipoor@gmail.com
}

Received 13 October 2012; revised 15 November 2012; accepted 24 November 2012

\begin{abstract}
Superparamagnetic magnetite nanoparticles (MNP) of about $10 \mathrm{~nm}$ were designed with proper physicochemical characteristics by an economic, biocompatible chemical co-precipitation of $\mathrm{Fe}^{2+}$ and $\mathrm{Fe}^{3+}$ in an ammonia solution, for hyperthermia applications. Synthetic methodology has been developed to get a well dispersed and homogeneous aqueous suspension of MNPs. Citric acid was used to stabilize the magnetite particle suspension, it was anchored on the surface of freshly prepared MNPs by direct addition method. Carboxylic acid terminal group not only render the particles more water dispersible but also provides a site for further surface modification. The naked MNPs are often insufficient for their stability, hydrophilicity and further functionalization. To overcome these limitations, citric acid was conjugated on the surface of the MNPs. The microstructure and morphology of the nanoparticles were characterized by X-ray diffraction (XRD) and transmission electron microscopy (TEM), and the interaction between citric acid and MNPs were characterized by Fourier transform infrared spectroscopy (FTIR), whereas the magnetic properties were investigated by vibrating sample magnetometry (VSM). Magnetic measurement revealed that the saturation magnetization of the nanoparticles was $74 \mathrm{emu} / \mathrm{g}$ and the nanoparticles were superparamagnetic at room temperature. We also have analyzed the potential of these particles for hyperthermia by determination of the specific absorption rate, the temperature increase $(\Delta T)$ of the particles was $37^{\circ} \mathrm{C}$. These ferrofluids with high self-heating capacity are a promising candidate for cancer hyperthermia treatment.
\end{abstract}

Keywords: Magnetite Nanoparticle; Citric Acid; Hyperthermia

\section{INTRODUCTION}

Superparamagnetic magnetite nanoparticles (MNPs) have received increased attention during the last decade due to their characteristic, i.e. inducible magnetic moments in the presence of an external magnetic field. Magnetite nanoparticles have been widely used experimentally for numerous biomedical and in vivo applications [1-3].

Magnetic-based delivery strategies are based on binding drugs with magnetic fluids that concentrate the drug in the site of interest. The surface-bound drugs can be released from the drug carriers by changing the physiological conditions, and are then taken up by the affected cells [4]. MNPs behave like magnets only in presence of an external magnetic field and do not retain any residual magnetism upon removal of the external magnetic field. This property makes it possible for their application as magnetic resonance imaging (MRI) contrast agent or their target ability under the influence of external magnets. However, MNPs are not stable at normal physiological conditions and show a tendency to aggregate because of the hydrophobic nature of these particles [5-7].

Challenges in fabricating and processing nanoparticles in regard to the applications in biomedicine are the issues of agglomeration, uniformity, hydrophilicity, and biocompatibility of nanoparticles. It is a technological challenge to acquire control over the nanoparticle sizes and dispersibility in desired solvents. Because of the large surface to volume ratio, nanoparticles possess high surface energies. The particles tend to aggregate to minimize total surface energy. In the case of metal oxide surfaces, such energies are in excess of $0.1 \mathrm{~N} / \mathrm{m}$ [8].

Suitable surface functionalization of the particles and choice of solvent are crucial to achieving sufficient repulsive interactions between particles to prevent aggregation and obtain a thermodynamically stable colloidal solution. The surface of MNPs can be stabilized in an aqueous dispersion by the adsorption of citric acid [9]. This process, as described by Sahoo et al. [9], occurs by the citric acid being coordinated via one or two of the carboxylate functionalities, depending upon steric necessity and the curvature of the surface. Carboxylates have important effects on the growth of iron oxide nanoparti- 
cles and their magnetic properties. Bee et al. demonstrated that the average diameter of citrate coated nanoparticles can be varied from $3 \mathrm{~nm}$ to $8 \mathrm{~nm}$ by decreasing the amount of citrate ions [10]. Liu and Huang have studied the effect of the presence of citric acid during iron oxide synthesis [11]. Increasing concentrations of citric acid caused significant decreases in the crystallinity of the iron oxides formed. Moreover, the presence of citrate led to changes in the surface geometry. The stability range is strongly dependent upon $\mathrm{pH}$ and the concentration of adsorbed acids [12].

Here we successfully capped citrate on the surface of superparamagnetic iron oxide nanoparticles as a hyperthermia device. The use of hyperthermia in the treatment of malignant tumors is as old as medicine itself. Hippocrates, the father of medicine, suggested that surface tumors should be cauterized by application of hot iron. In modern times, more advanced methods (hot water bath, high-frequency radiation and magnetic fluid hyperthermia) are employed to heat, and hopefully destroy tumors [13].

Nowadays, there is a great interest for nano-thermotherapy, i.e. killing tumor cells, not with chemotherapeutic agents, but with locally targeted nano-heaters that would basically kill malignant cells [14]. This technique is an encouraging technique for cancer treatment because of ease of targeting the cancerous tissue, thus having fewer side effects than radiotherapy and chemotherapy. Moreover, this method provides a straight forward way of treating cancer in combination with irradiation and chemotherapy.

In this study we focused our interest on the physicochemical aspects of the particles. Finally, the heating properties of these nanoparticles were evaluated, showing potential for hyperthermia.

\section{MATERIALS AND METHODS}

\subsection{Materials}

Ferric chloride hexahydrate, Ferrous chloride tetrahydrate, Citric acid (CA) and Ammonia solution (25\%) were purchased from Merck (Germany). All chemicals were of analytical grade and used as received.

\subsection{Magnetite Nanoparticle Synthesis}

The preparation of magnetite nanoparticles was performed by a chemical coprecipitation of $\mathrm{Fe}^{2+}$ and $\mathrm{Fe}^{3+}$ ions $(1: 2$ molar ratio) by the addition of $\mathrm{NH}_{4} \mathrm{OH}$. Briefly, $50 \mathrm{ml}$ of $1.0 \mathrm{~mol} / \mathrm{L} \mathrm{Fe}^{2+}$ and $2.0 \mathrm{~mol} / \mathrm{L} \mathrm{Fe}^{3+}$ solutions were prepared with deionized water in two beakers, and then transferred to a three necked flask and temperature was slowly increased to $80^{\circ} \mathrm{C}$ in refluxing condition under nitrogen atmosphere. While vigorously stirring the reac- tion mixture to prevent the development of large polycrystalline particles, turbulence was created by placing the reaction flask in an ultrasonic bath $(100 \mathrm{~W})$ and controlling the homogenization (overhead stirrer) rate (9000 rpm) during the initial two minutes of the reaction. $\mathrm{NH}_{4} \mathrm{OH}(25 \mathrm{wt} \%)$ was added instantaneously to the reaction mixture and vigorous mechanical stirring was applied to reach $\mathrm{pH} 10-11$.

The surface of MNPs was stabilized with citric acid by a direct addition method, to obtain modified MNPs with carboxylic groups. Briefly, the MNPs surface were treated with citrate ions by incubating for 1 hour within 0.5 $\mathrm{g} / \mathrm{mL}$ citric acid solution and reaction temperature was raised up to $90^{\circ} \mathrm{C}$ and the reaction was completed for 60 min with continuous stirring. The black precipitates were acquired by cooling the reaction mixture to room temperature. At last the suspensions were washed several times with deionized water. The particles did not settle down under the influence of magnet, as a result of stability of solution. The as-formed reaction product contained an excess of citric acid and so, the nanoparticle dispersion was centrifuged and washed several times. Magnetite nanoparticles stabilized with citric acid are abbreviated as MNP-CA.

\subsection{Characterization of the Nanoparticles}

The synthesized nanoparticles were characterized by various analytical techniques. The X-ray diffraction (XRD) patterns were acquired from dried nanoparticle samples with a BRUKER X-ray powder diffractometer by using $\mathrm{Cu}-\mathrm{K}_{\alpha 1}(1.54060 \AA)$ radiation. The scans of selected diffraction peaks were carried out in step mode (step size $0.02^{\circ}$, measurement time $2 \mathrm{~s}$, measurement temperature $300 \mathrm{~K}$ and standard: Si powder).

Transmission electron microscopy (TEM) images were acquired on a Phillips 400 TEM operating at 100 $\mathrm{kV}$. TEM grids were prepared by depositing a drop of diluted nanoparticle suspension on 300 mesh siliconmonoxide support films and drying the grids under vacuum for $2 \mathrm{~h}$.

The infrared spectra were recorded in the range 400 $4000 \mathrm{~cm}^{-1}$ on a Fourier transform infrared spectrometer (FTIR-Shimadzu-8000). Samples of the surface modified nanoparticles were dried overnight using a Virtis Freezemobile freeze-drier. Nanoparticle powder (2 mg) was then milled with $\mathrm{KBr}$ and the mixture was pressed into a pellet for analysis.

The saturation magnetization $\left(\mathrm{M}_{\mathrm{s}}\right)$ and coercive force $\left(\mathrm{H}_{\mathrm{c}}\right)$ of the samples were measured using a vibrating sample magnetometer (VSM, Dexing, Model: 250) with a sensitivity of $10^{-3}$ emu. The magnetic field was changed uniformly with a time rate of $66 \mathrm{Oe} / \mathrm{s}$.

In order to test the heating capacity of the MNPs, we 
have prepared samples of the synthesized MNP-CA suspended in distilled water with a magnetic composition of $1 \% \mathrm{w} / \mathrm{v}$ ( $\mathrm{g}$ of $\mathrm{Fe}_{3} \mathrm{O}_{4} / \mathrm{mL}$ solution). These samples were placed in a magnetic field with a constant frequency of $215 \mathrm{kHz}$ and magnetic field amplitude of $3 \mathrm{kAm}^{-1}$ for a period of $20 \mathrm{~min}$. The temperature of the surrounding medium was kept constant using a heated chamber at $25^{\circ} \mathrm{C}$ and the temperature was measured by infrared (IR) thermal camera under an alternating magnetic field.

\section{RESULTS AND DISCUSSION}

Figure 1 shows the process of the surface modification of suoerparamagnetic magnetite nanoparticles. MPNs were synthesized and then stabilized by citric acid.

\subsection{XRD Analysis}

Figure 2 shows the XRD pattern of MNPs which indicates a highly crystalline cubic spinel structure. The reflection peak positions and relative intensities of MNPs agree well with the XRD pattern of magnetite in the literature, which confirms the structure of the magnetite [15].

\subsection{TEM Analysis}

Figure 3 presents the typical TEM image and size distribution of as-prepared samples. As shown in Figure 3, MNPs were about $10 \mathrm{~nm}$ in average size with spherical morphology and narrow size distribution.

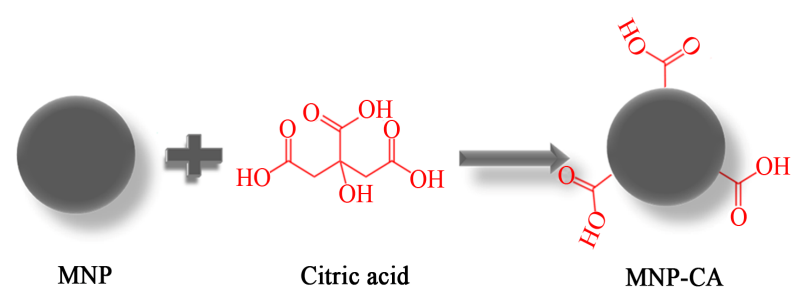

Figure 1. Scheme for the preparation of magnetic nanoparticles (MNP) by citric acid (CA).

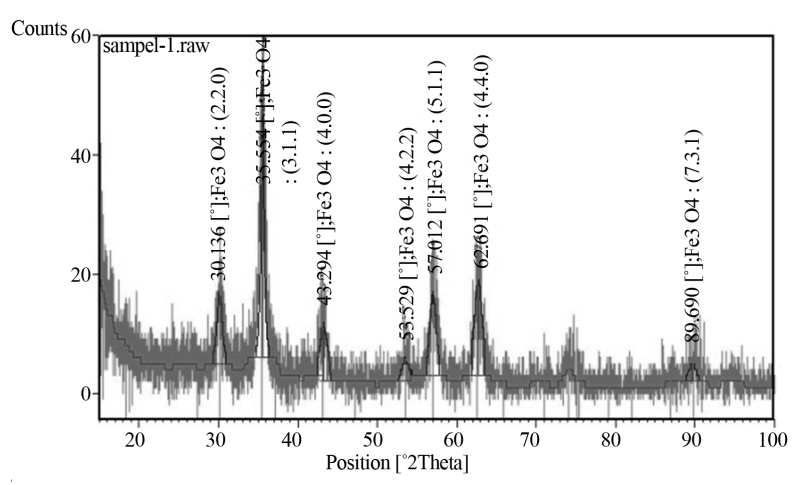

Figure 2. XRD pattern of MNP shows the composition and crystal structure of magnetite.

\subsection{FTIR Analysis}

Figure 4 shows the FT-IR spectra of pure citric acid (CA), citrate stabilized MNP.

The absorption bands for pure CA are resolved, but those of the MNP-CA are broad and few. In Figure 4, an intense band at $3300 \mathrm{~cm}^{-1}$ proposes the presence of nondissociated $\mathrm{OH}$ groups of citric acid. The peak around $2940 \mathrm{~cm}^{-1}$ is due to $\mathrm{CH}_{2}$ stretching, peak at $1646 \mathrm{~cm}^{-1}$ may be assigned to the symmetric stretching of $\mathrm{OH}$ from $\mathrm{COOH}$ group, displaying the binding of a citric acid radical to the magnetite surface. The $1755 \mathrm{~cm}^{-1}$ peak of $\mathrm{CA}$, attributable to the $\mathrm{C}=\mathrm{O}$ vibration from the $\mathrm{COOH}$ group of CA. This peak shifts to an intense band at about $1646 \mathrm{~cm}^{-1}$ for MNP-CA, displaying the binding of a CA radical to the surface of $\mathrm{Fe}_{3} \mathrm{O}_{4}$ nanoparticles by chemisorptions of carboxylate (citrate) ions [16-18]. Carboxylate groups of $\mathrm{CA}$ form complexes with $\mathrm{Fe}$ atoms on the surface of $\mathrm{Fe}_{3} \mathrm{O}_{4}$ representing partial single bond character to the $\mathrm{C}=\mathrm{O}$ bond. The next band at $1429 \mathrm{~cm}^{-1}$ can be allocated to the asymmetric stretching of $\mathrm{CO}$ from $\mathrm{COOH}$ group. The strong FTIR band observed at around $520 \mathrm{~cm}^{-1}$ can be attributed to the $\mathrm{Fe}-\mathrm{O}$ stretching vibration mode of $\mathrm{Fe}_{3} \mathrm{O}_{4}$ [19]. Therefore, we could say that the citric acid binds to the magnetite surface by carboxylate.

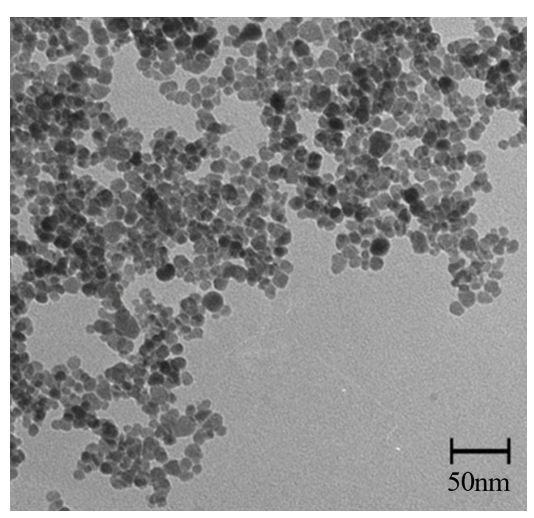

Figure 3. TEM micrograph of MNP-CA.

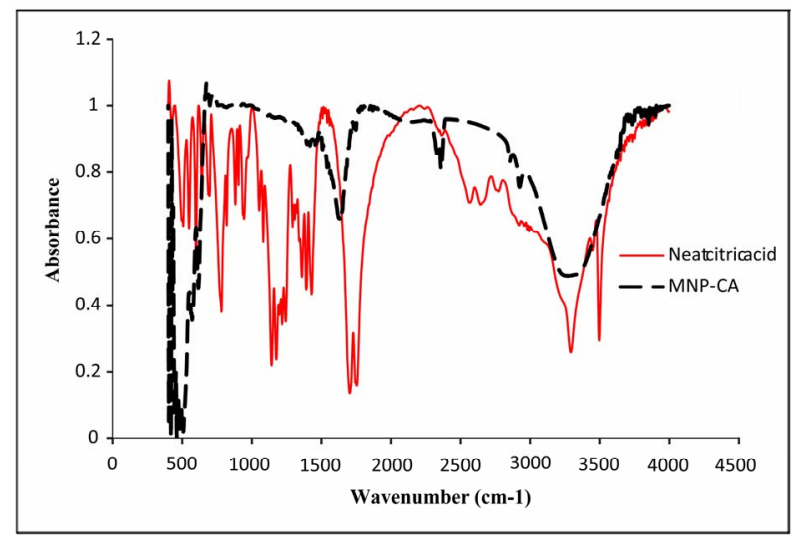

Figure 4. FTIR of neat CA and MNP-CA. 


\subsection{VSM Analysis}

Figure 5 shows VSM plot of MNP-CA at $300 \mathrm{~K}$. The hysteresis loop does not display magnetic remanence, and thus the nanoparticles are considered to be superparamagnetic. The magnetization saturation $\left(\mathrm{M}_{\mathrm{s}}\right)$ of the MNPs was $74 \mathrm{emu} / \mathrm{g}$. For biomedical applications such as hyperthermia, it is required that the nanoparticles have high saturation magnetization, uniform particle size and to be superparamagnetic. Therefore, these ferrofluids that have high magnetic response, could be used for hyperthermia treatment.

\subsection{Heating Study}

Our formulations were tested for heating effects and results are presented in Figure 6. The temperature of formulations is plotted as a function of time at the constant frequency of $215 \mathrm{kHz}$ and magnetic field amplitude of 3 $\mathrm{kAm}^{-1}$.

The main parameter determining the heating of the tissue is the specific absorption rate (SAR). It is ex-

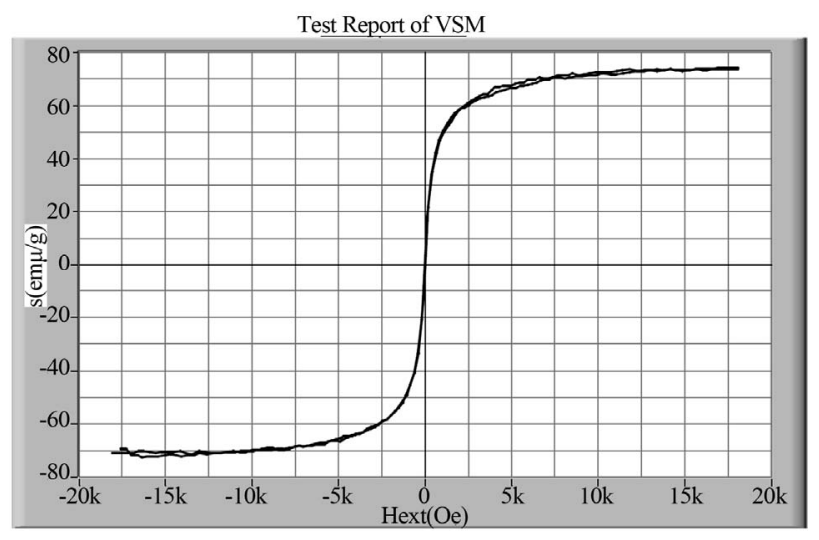

Figure 5. Vibrating sample magnetometry (VSM) of MNP-CA.

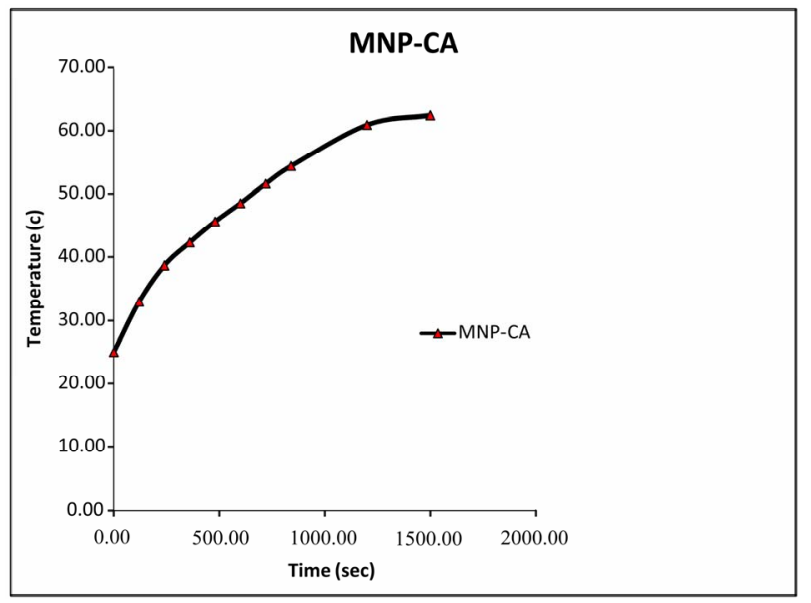

Figure 6. Temperature as a function of time for MNP-CA suspended in distilled water under alternating magnetic field of $215 \mathrm{kHz}$. pressed in Watt per kilogram. The SAR formula (Equation (1)) for the adiabatic case is [20]:

$$
\mathrm{SAR}=\frac{\Delta \mathrm{T} \mathrm{C}}{\Delta \mathrm{t} \mathrm{m}_{\mathrm{fe}}}
$$

where $\mathrm{C}$, is the specific heat of solvent, dT/dt is the initial slope of the time dependent temperature curve and $\mathrm{m}_{\mathrm{fe}}$ is mass fraction of $\mathrm{Fe}$ in the sample. To obtain an accurate SAR value, an adiabatic environment is assumed, where the initial temperature of the sample must be equal to the temperature of the surrounding medium. In our experimental setup, a sample of the functionalized MNPs was placed in an isolated environment. The temperature of the surrounding medium was kept constant using a heated chamber at $25^{\circ} \mathrm{C}$. We used Equation (1) to calculate the SAR of the prepared magnetic samples assuming the heat capacity of these diluted samples as the heat capacity of water $\left(\mathrm{C}=\mathrm{C}_{\text {water }}=4.18 \mathrm{~J} / \mathrm{g}^{\circ} \mathrm{C}\right)$. For magnetite nanoparticles, we have calculated a SAR of about $16.74 \mathrm{w} / \mathrm{g}$. The reason for the high value of SAR in MNP-CA Samples (16.74 w/g) was the small particle size of these particles.

As shown in Figure 6, the high temperature change of $37^{\circ} \mathrm{C}$ was measured in MNP-CA suspension.

\section{CONCLUSION}

MNPs were synthesized by co-precipitation method and then capped with citric acid to render particles with reactive carboxyl groups on their surface. Eventually, due to the small sizes of the nanoparticles and the superparamagnetic behavior of the particles demonstrated here should be of notable value in numerous areas of biomedical research. Citrate capped MNPs had remarkable heating effect during the application of a magnetic field, which make them attractive for biomedical heating applications, such as magnetic fluid hyperthermia.

\section{REFERENCES}

[1] Dandamudi, S. and Robert B.C. (2007) The drug loading, cytotoxicty and tumor vascular targeting characteristics of magnetite in magnetic drug targeting. Biomaterials, 28, 4673-4683. doi:10.1016/j.biomaterials.2007.07.024

[2] Li, Z., Li, M., Kawashita, Araki, N., Mitsumori, M., Hiraoka, M. and Doi, M. (2010) Magnetite nanoparticles with high heating efficiencies for application in the hyperthermia of cancer. Materials Science and Engineering: C, 30, 990-996. doi:10.1016/j.msec.2010.04.016

[3] Wang, Y., Xu, F., Zhang, C., Lei, D., Tang, Y., Xu, H., Zhang, Z., Lu, H., Du, X. and Yang, G. (2011) MR high, sensitive fluorescent magnetite nanocluster for stem cell tracking in ischemic mouse brain. Nanomedicine: Nanotechnology, Biology and Medicine, 6, 1009-1019. doi:10.1016/j.nano.2011.03.006

[4] Parveen, S., Misra, R. and Sahoo, S.K. (2011) A boon to 
drug delivery, therapeutics, diagnostics and imaging. Nanomedicine, Nanotechnology, Biology, and Medicine, 2 , 147-166.

[5] Mahmoudi, M., Simchi, A. and Imani, M. (2010) Recent advances in surface engineering of superparamagnetic iron oxide nanoparticles for biomedical applications. Journal of the Iranian Chemical Society, 7, 1-27.

[6] Mornet, S., Portier, J. and Duguet, E. (2005) A method for synthesis and functionalization of ultrasmall superparamagnetic covalent carriers based on maghemite and dextran. Journal of Magnetism and Magnetic Materials, 293, 127-134. doi:10.1016/j.jmmm.2005.01.053

[7] Jain, T.K., Morales, M.A., Sahoo, S.K., Leslie-Pelecky, D.L. and Labhasetwar, V. (2005) Iron oxide nanoparticles for sustained delivery of anticancer agents. Molecular Pharmaceutics, 2, 194-205. doi:10.1021/mp0500014

[8] Vayssieres, L., Chaneac, C., Troc, E. and Jolivet, J.P. (1998) Size tailoring of magnetite particles formed by aqueous precipitation: An example of thermodynamic stability of nanometric oxide particles. The Journal of Colloid and Interface Science, 205, 205-205. doi:10.1006/jcis.1998.5614

[9] Sahoo, Y., Goodarzi, A., Swihart, M.T., Ohulchanskyy, T.Y., Kaur, N., Furlani, E.P. and Prasad, P.N. (2005) Aqueous ferrofluid of magnetite nanoparticles: Fluorescence labeling and magnetophoretic control. Australian Journal of Chemistry B, 109, 3879-3885. doi:10.1021/jp045402y

[10] Liu, C. and Huang, P.M. (1999) Atomic force microscopy and surface characteristic of iron oxides formed in citrate solution. Soil Science Society of America Journal, 63, 65-72. doi:10.2136/sssaj1999.03615995006300010011x

[11] Laurent, S., Forge, D., Port, M., Roch, A., Robic, C., Vander Elst, L. and Muller, R. (2008) Magnetic iron oxide nanoparticles: Synthesis, stabilization, vectorization, physicochemical characterizations and biological applications. Chemistry Reviews, 108, 2064-2110. doi:10.1021/cr068445e

[12] Bee, A., Massart, R. and Neveu, S. (1995) Synthesis of very fine maghemite particles. Journal of Magnetism and Magnetic Materials, 149, 6-9. doi:10.1016/0304-8853(95)00317-7

[13] Nielsen, O.S., Horsman, M. and Overgaard, J. (2001) A future for hyperthermia in cancer treatment. European Journal of Cancer, 37, 1587-1589. doi:10.1016/S0959-8049(01)00193-9

[14] Gazeau, F., Levy, M. and Wilhelm, C. (2008) Optimizing magnetic nanoparticle design for nanothermotherapy. $\mathrm{Na}$ nomedicine, 3, 831-844. doi:10.2217/17435889.3.6.831

[15] Levy, L., Sahoo, Y., Kim, K., Bergey, E.J. and Prasad, P.N. (2002) Nanochemistry: Synthesis and characterization of multifunctional nanoclinics for biological applications. Chemistry of Materials, 14, 3715-3720. doi:10.1021/cm0203013

[16] Sahoo, Y., Goodarzi, A., Swihart, M.T., Ohulchanskyy, T.Y., Kaur, N., Furlani, E.P. and Prasad, P.N. (2005) Aqueous ferrofluid of magnetite nanoparticles: Fluorescence labeling and magnetophoretic control. Australian Journal of Chemistry B, 109, 3879-3885. doi:10.1021/jp045402y

[17] Nigam, S., Barick, K.C. and Bahadur, D. (2011) Development of citrate-stabilized $\mathrm{Fe}_{3} \mathrm{O}_{4}$ nanoparticles: Conjugation and release of doxorubicin for the rapeuticapplications. Journal of Magnetism and Magnetic Materials, 323, 237-243. doi:10.1016/j.jmmm.2010.09.009

[18] Racuciu, M., Creanga, D.E. and Airinei, A. (2006) Citric-acid-coated magnetite nanoparticles for biological applications. European Physical Journal E (EPJE), 21, 117121. doi:10.1140/epje/i2006-10051-y

[19] Levy, L., Sahoo, Y., Kim, K., Bergey, E.J. and Prasad, P.N. (2002) Nanochemistry: Synthesis and characterization of multifunctional nanoclinics for biological applications. Chemistry of Materials, 14, 3715-3720. doi:10.1021/cm0203013

[20] Giri, J., Pradhan, P., Sriharsha, T. and Bahadur, D. (2005) Preparation and investigation of potentiality of different soft ferrites for hyperthermia applications. Journal of Applied Physics, 97, 910-916. doi:10.1063/1.1855131 